\title{
Circuit
}

Musiques contemporaines

\section{Word Events : Perspectives on Verbal Notation, de John Lely et James Saunders, New York ; Londres, Continuum, 2012, 456 pages}

\section{Cléo Palacio-Quintin}

Volume 25, numéro 1, 2015

URI : https://id.erudit.org/iderudit/1029480ar

DOI : https://doi.org/10.7202/1029480ar

Aller au sommaire du numéro

Éditeur(s)

Les Presses de l’Université de Montréal

ISSN

1183-1693 (imprimé)

1488-9692 (numérique)

Découvrir la revue

Citer ce compte rendu

Palacio-Quintin, C. (2015). Compte rendu de [Word Events : Perspectives on

Verbal Notation, de John Lely et James Saunders, New York ; Londres,

Continuum, 2012, 456 pages]. Circuit, 25(1), 81-83.

https://doi.org/10.7202/1029480ar

Ce document est protégé par la loi sur le droit d'auteur. L'utilisation des services d'Érudit (y compris la reproduction) est assujettie à sa politique d'utilisation que vous pouvez consulter en ligne.

https://apropos.erudit.org/fr/usagers/politique-dutilisation/
Cet article est diffusé et préservé par Érudit.

Érudit est un consortium interuniversitaire sans but lucratif composé de l’Université de Montréal, l'Université Laval et l'Université du Québec à Montréal. Il a pour mission la promotion et la valorisation de la recherche. https://www.erudit.org/fr/ 


\section{Word Events: Perspectives on Verbal Notation, de John Lely et James Saunders}

New York; Londres, Continuum, 2012, 456 pages.

Compte rendu de Cléo Palacio-Quintin

La notation verbale, c'est-à-dire des œuvres/performances dont les instructions de réalisation sont données sous forme de texte, est une pratique artistique assez répandue depuis la fin des années 1950, mais qui a été jusqu'ici assez peu documentée. Après avoir effectué des recherches sur plus de 2500 textes du genre, les compositeurs John Lely ${ }^{1}$ et James Saunders ${ }^{2}$ remédient à cette situation en publiant un imposant ouvrage au sujet de ces «partitions » données sous forme de texte.

Leur plus importante contribution est certainement d'avoir réuni plus de 150 partitions verbales dans une seule publication, allant des plus connues, voire «classiques» de George Brecht, Alvin Lucier, Karlheinz Stockhausen et La Monte Young, à de plus récentes, mais non moins fascinantes, de Seth Kim-Cohen, Michael Pisaro ou Jennifer Walshe, pour n'en citer que quelques-unes. Le lecteur se trouve donc devant une incroyable panoplie d'œuvres conceptuelles, tout aussi captivantes pour l'analyste-musicologue que pour le compositeur ou l'interprète en quête d'inspiration. La consultation de certaines pages génère même la réalisation de la performance comme telle.

Grand nombre de ces œuvres prescrivent de créer de la musique, mais les instructions sont accessibles à tout lecteur et non seulement au musicien qui connaît

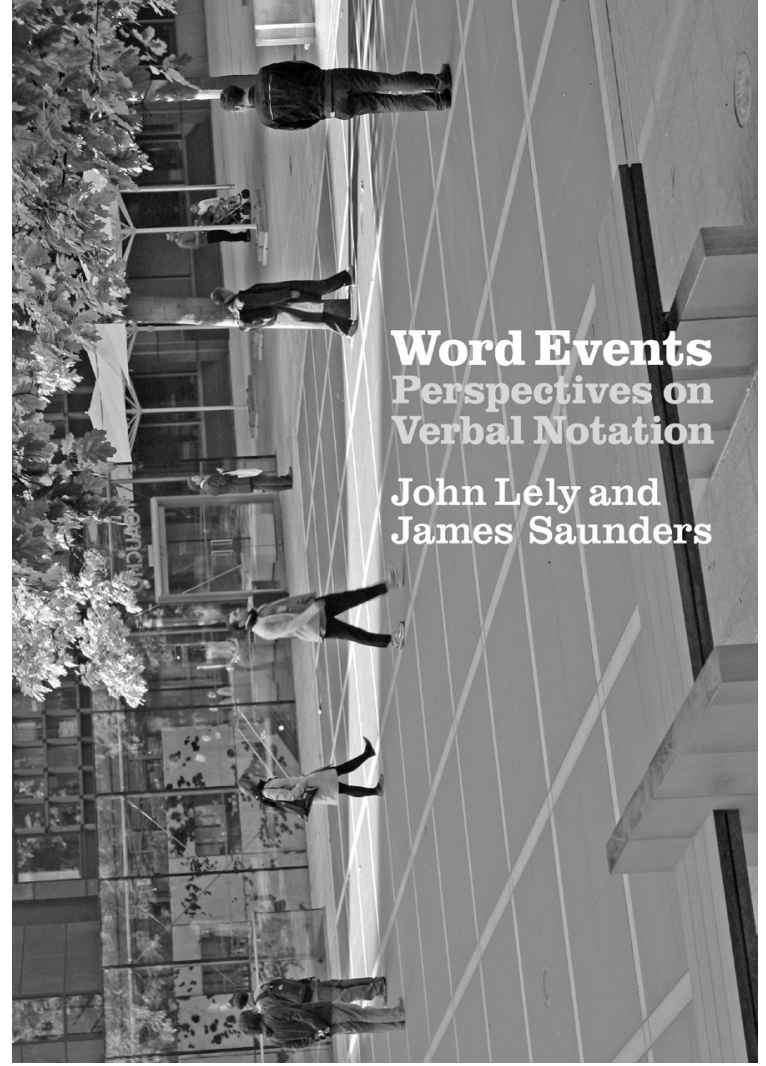

la notation musicale traditionnelle. Par exemple, The Great Learning (1968-1971) de Cornelius Cardew (p. 149) s'adresse autant à des musiciens qu'à des nonmusiciens, puisqu'aucune notion musicale n'est nécessaire pour comprendre les instructions de jeu. D'autres partitions font appel à des performances de nature plutôt conceptuelle et parfois théâtrale - formes prédominantes dans les Events de Fluxus - ou permettent de 
réaliser des œuvres visuelles. Un bel exemple est celui du Wall Drawing \#96o (2001) de Sol LeWitt (p. 244), qui, avec une seule phrase d'instructions, permet de recréer une murale complète authentifiée par l'artiste: "A straight line about 18" $45.7 \mathrm{~cm})$ long is drawn; from its midpoint, another line about 18" $(45.7 \mathrm{~cm})$ long; from the midpoint of each subsequent line, another line 18" $(45.7 \mathrm{~cm})$ long, uniformly dispersed covering the entire surface of the wall. » Lœuvre consiste donc non seulement en son résultat (la murale sera visuellement toujours relativement semblable), mais aussi en tout son processus de réalisation.

Le livre est divisé en deux grandes parties très distinctes, à la fois en contenu et en volume. La première propose une intéressante approche d'analyse grammaticale des textes de notation verbale $(71 \mathrm{p}$.$) ,$ alors que la deuxième consiste en un recueil des partitions, accompagnées de commentaires et d'analyses des compositeurs eux-mêmes ou écrites par d'autres auteurs (36o p.). Le livre contient également une table des matières très détaillée ainsi que la liste des illustrations (la plupart des partitions étant présentées en fac-similé), une description des sources et une riche bibliographie, en plus d'un index très utile pour butiner ici et là dans cette masse d'informations afin d'y trouver des compositeurs ou sujets qui nous interpellent plus particulièrement.

La première partie, rédigée par John Lely et intitulée "On the Use of Grammar in Verbal Notation", apporte un éclairage fascinant sur les différentes perspectives suggérées par la grammaire employée dans ce type de texte. L'auteur a étudié un grand corpus d'œuvres en s'appuyant sur la grammaire fonctionnelle systémique ${ }^{3}$, une approche descriptive qui analyse comment le langage est utilisé pour interpréter la réalité, faire des choix et adopter des relations sociales. Comme son nom l'indique, cette méthode repose sur la fonction des choix grammaticaux dans l'usage lorsqu'appliquée aux partitions verbales. Lely en fait une excellente démonstration en expliquant chacun des domaines grammaticaux - contexte, registre, processus, temps, modalité, humeur, voix, circonstances - à l'aide d'exemples concrets, tirés de différentes partitions. Ses analyses et commentaires nous font percevoir, avec beaucoup plus de précision et de nuances, les intentions des compositeurs dans leurs instructions, souvent assez brèves, mais qui finalement contiennent beaucoup plus d'informations que l'on ne l'imagine à la première lecture.

La deuxième partie du livre regroupe une panoplie de partitions composées entre 1959 et 2010, par une cinquantaine d'artistes. Les partitions sont très variées, autant dans leur facture que dans leur possible résultat (sonore ou non). Chaque œuvre est accompagnée d'un ou deux textes, souvent des commentaires écrits pour l'occasion par les artistes eux-mêmes ${ }^{4}$. Lely et Saunders rédigent certains des commentaires et partagent aussi des expériences de leurs propres réalisations de quelques pièces, ainsi que des entretiens réalisés avec d'autres interprètes. Ce livre permet aussi de republier d'excellents écrits plus anciens d'artistes comme Brecht, Cardew, Lucier, Wolff et La Monte Young.

Les partitions sont simplement présentées par ordre alphabétique des noms des créateurs, un peu sous forme de catalogue, sans perspective historique ou regroupements par type de notation ou d'approche conceptuelle. Chaque œuvre est donc présentée pour elle-même et les commentaires des créateurs sont souvent assez personnels. Ce sera au lecteur d'établir des relations dans ce corpus éclectique, qu'il pourra consulter dans le désordre, comme bon lui semble. Heureusement, certains des commentaires rédigés par Lely et Saunders font souvent référence à d'autres œuvres et donnent une perspective historique en situant la pièce à la fois dans la production de l'artiste et dans le contexte artistique de sa réalisation. La 
plupart des textes sont très éclairants et inspirants, et les multiples auteurs et points de vue exposés donnent ainsi une très large perspective sur la notation verbale sous toutes ses formes.

Nous aurions souhaité retrouver encore plus d'analyses grammaticales de Lely dans cette deuxième partie du livre, qui fait peu écho à la première partie plus théorique, mais qui est tout aussi captivante. Il demeure que cette publication est une ressource précieuse, une référence à consulter pour quiconque s'intéresse au sujet ou souhaite le découvrir. Les interprètes souhaitant expérimenter de nouvelles formes de performance seront choyés par l'éventail des découvertes et tout compositeur y trouvera certainement matière à réflexion.

1. Sur John Lely, voir: <www.johnlely.co.uk> (consulté le 26 janvier 2015).

2. John Saunders dirige le Centre for Musical Research de l'Université Bath Spa au Royaume-Uni, où s'est effectuée la recherche ayant mené à cette publication. Voir : <www.james-saunders.com> (consulté le 26 janvier 2015).

3. La Systemic Functional Grammar, ou SFG, est une approche grammaticale sémiotique développée par Michael Halliday.

4. Certains compositeurs/artistes en ont aussi profité pour réviser leur œuvre dans le cadre de cette publication.

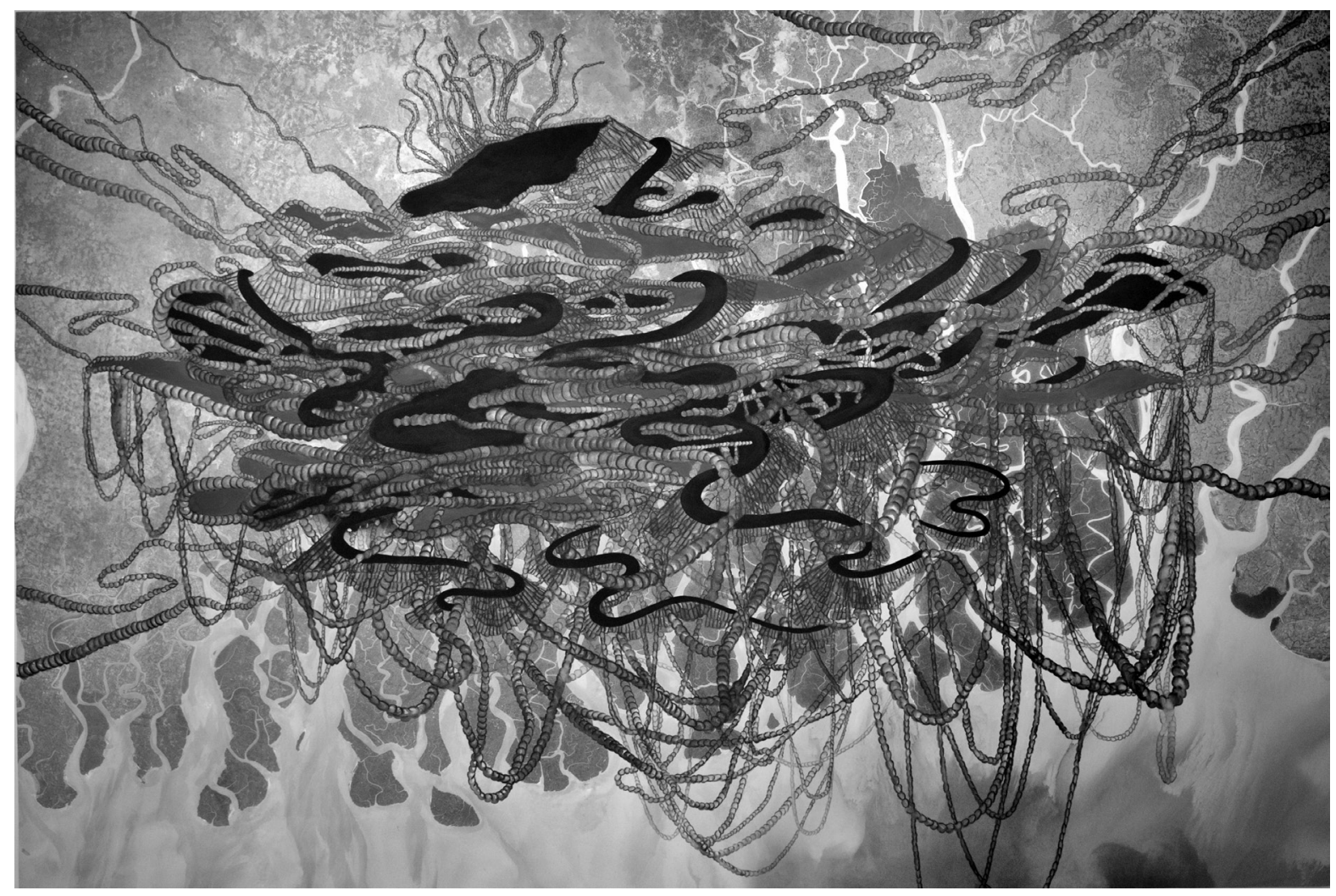

Catherine Bolduc, South Talpatti en lévitation au-dessus des Sundarbans, 2013. Impression numérique, peinture acrylique et aquarelle sur papier, 122 x $183 \mathrm{~cm}$. Collection de la Banque Nationale. 